\title{
Development and validation of a nutrition knowledge questionnaire for a Canadian population
}

Maude Bradette-Laplante ${ }^{1,2}$, Élise Carbonneau ${ }^{1,2}$, Véronique Provencher ${ }^{1,2}$, Catherine Bégin $^{1,3}$, Julie Robitaille ${ }^{1,2}$, Sophie Desroches ${ }^{1,2}$, Marie-Claude Vohl ${ }^{1,2}$, Louise Corneau ${ }^{1,2}$ and Simone Lemieux ${ }^{1,2, *}$

'Institute of Nutrition and Functional Foods, Laval University, 2440 boulevard Hochelaga, Québec, QC, Canada, GIV OA6: ${ }^{2}$ School of Nutrition, Laval University, Québec, QC, Canada: ${ }^{3}$ School of Psychology, Laval University, Québec, QC, Canada

Submitted 1 June 2016: Final revision received 11 November 2016: Accepted 21 November 2016: First published online 27 December 2016

\begin{abstract}
Objective: The present study aimed to develop and validate a nutrition knowledge questionnaire in a sample of French Canadians from the province of Quebec, taking into account dietary guidelines.

Design: A thirty-eight-item questionnaire was developed by the research team and evaluated for content validity by an expert panel, and then administered to respondents. Face validity and construct validity were measured in a pre-test. Exploratory factor analysis and covariance structure analysis were performed to verify the structure of the questionnaire and identify problematic items. Internal consistency and test-retest reliability were evaluated through a validation study.

Setting: Online survey.

Subjects: Six nutrition and psychology experts, fifteen registered dietitians (RD) and 180 lay people participated.

Results: Content validity evaluation resulted in the removal of two items and reformulation of one item. Following face validity, one item was reformulated. Construct validity was found to be adequate, with higher scores for RD $v$. non-RD (21.5 (SD 2.1) $v .15 \cdot 7$ (SD 3.0) out of $24, P<0.001$ ). Exploratory factor analysis revealed that the questionnaire contained only one factor. Covariance structure analysis led to removal of sixteen items. Internal consistency for the overall questionnaire was adequate (Cronbach's $\alpha=0.73$ ). Assessment of test-retest reliability resulted in significant associations for the total knowledge score $(r=0.59, P<0 \cdot 001)$.

Conclusions: This nutrition knowledge questionnaire was found to be a suitable instrument which can be used to measure levels of nutrition knowledge in a Canadian population. It could also serve as a model for the development of similar instruments in other populations.
\end{abstract}

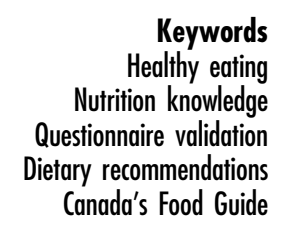

Healthy eating Nutrition knowledge Questionnaire validation Canada's Food Guide
Food intake has been investigated widely and found to be determined by a combination of multiple factors on different levels, namely individual, social and environmental ${ }^{(1)}$. Among individual determinants, nutrition knowledge is considered as one of the factors affecting food intake ${ }^{(2)}$. A recent systematic review reported a significant positive association between high nutrition knowledge and consumption of fruits and vegetables ${ }^{(3)}$. Since it has been shown in the literature that a high intake of fruits and vegetables is an excellent indicator of a healthy diet ${ }^{(4,5)}$, this suggests that a high level of nutrition knowledge would be linked with overall healthy food intakes. Improving nutrition knowledge can also favour the development of healthier eating behaviours. For instance, it was recently demonstrated that greater nutrition knowledge is linked with higher odds of engaging in healthy weight-loss behaviours ${ }^{(6)}$. Nutrition knowledge also plays an important role in public health campaigns promoting healthy eating. In fact, in most of these campaigns, improving nutrition knowledge is a target to favour better dietary intakes within the population ${ }^{(3)}$. In that context, nutrition knowledge is therefore an important indicator to measure the impact of these public health interventions $^{(7-9)}$.

In view of the importance of assessing nutrition knowledge in nutrition education programmes and in other contexts, it appears essential to use questionnaires that accurately evaluate the constructs intended to be measured. The relevance of valid instruments for measurement 
of nutrition knowledge has been raised as a key aspect to ensure legitimacy of results obtained from studies using nutrition knowledge as a determinant of food intake. It has even been shown that when thoroughly validated questionnaires are used to measure nutrition knowledge, positive associations are more likely to be observed between nutrition knowledge and diet quality, which reinforces the importance of the validation process ${ }^{(3)}$.

Parmenter and Wardle ${ }^{(10)}$ are the instigators of the General Nutrition Knowledge Questionnaire (GNKQ), which was validated with a variety of methods to ensure its accuracy. Some studies have used a modified version of the GNKQ adapted to their population and observed that the differences in recommendations between their own food guide and the one from the GNKQ necessitated many modifications, which affected the validity of the modified instruments $^{(11,12)}$. According to Parmenter and Wardle ${ }^{(13)}$, a new instrument should be developed if no existing questionnaire relevant to the particular study can be found. In the context of the present research, the questionnaire was to be used in a study to evaluate whether knowledge of the latest 2007 version of Canada's Food Guide $(\mathrm{CFG})^{(14)}$ was associated with actual adherence to $\mathrm{CFG}$ recommendations. CFG is a tool designed by the Canadian government to translate the science of nutrition into a healthy eating pattern adapted to the Canadian population's reality. It has the shape of a rainbow and is divided into four food groups (Vegetables and fruits, Grain products, Milk and alternatives, Meat and alternatives), including recommended numbers of servings from each food group for each sex and age category. CFG also includes specific guidelines for each food group to help consumers make most of their servings. This tool is intended for healthy Canadians and is readily available to the population, particularly in schools. Most Canadians (86.5\%) report having general awareness of $\mathrm{CFG}^{(15)}$. For the design of a questionnaire related to $\mathrm{CFG}$ recommendations, specific items were required. To our knowledge, no such questionnaire exists and a new questionnaire was necessary to assess nutrition knowledge in a Canadian context.

Therefore the purpose of the present study was to develop and validate a nutrition knowledge questionnaire specifically designed for a French-Canadian population. More precisely, the study aimed to design a questionnaire based on literature and existing questionnaires to evaluate mainly knowledge of CFG, but also general nutrition knowledge, and measure both its validity and reliability, using several validation methods, in a French-Canadian sample from the province of Quebec.

\section{Methods}

\section{Development of the items}

The steps proposed by Parmenter and Wardle ${ }^{(13)}$ were used for guidance in the design of the questionnaire, ensuring a valid method of development of the items. The first step was to define the scope of the measure and included ensuring that the items assessed knowledge rather than beliefs. The second step was generation of the items, including reviewing the literature, nutrition information documents and Canadian surveys on food and nutrition, to create items included in the questionnaire. For the third step, as suggested by Parmenter and Wardle ${ }^{(13)}$, a sample of people similar to the main sample completed the questionnaire, and we named this step the pre-test in our study. The fourth step, assessment of reliability, was performed through different methods such as test-retest reliability. The last step was assessing validity of the instrument, namely by evaluating content and construct validity.

The aim of the questionnaire was to assess nutrition knowledge using the concept of healthy eating as seen in the CFG and in recent guidelines ${ }^{(16)}$. Based on previous questionnaires assessing the topic, two specific areas of knowledge most adapted to this questionnaire were identified a priori: (i) familiarity with CFG (e.g. food groups, portions, guidelines); and (ii) general nutrition knowledge (e.g. knowledge about foods, food-disease relationships).

\section{Items, design and format of the questionnaire}

Questionnaire items were developed by a registered dietitian (RD) and a researcher in the field of nutrition. 'Familiarity with CFG' items were designed using existing questionnaires and surveys for the Canadian population on food and nutrition ${ }^{(17,18)}$. Items were created to cover different aspects of the CFG, namely food groups, portions and specific guidelines. A total of twenty-five items were developed for this section. Four items on knowledge of number of portions recommended for each food group were assessed with a short open-ended question (e.g. 'How many portions a day do you think CFG recommends, for an individual of your age and gender, for each of the following food groups?'). Four multiple-choice items were included to assess knowledge of the amount of food in a portion of the different groups of the CFG (e.g. 'To how many CFG portions do you think the following food quantities correspond?'). A series of ten 'yes' or 'no' items assessed knowledge of specific guidelines from the CFG (e.g. 'Indicate whether the following statements are included in CFG recommendations: Eat at least one green vegetable every day'). For each item, an 'I don't know' option was available, as it has been shown to remove pressure from participants and also to ensure answers are not randomly assigned ${ }^{(13)}$. One multiple-choice item assessed understanding of the meaning of CFG.

'General nutrition knowledge' items were developed using existing questionnaires ${ }^{(19-21)}$. An initial pool of thirteen items was developed to address either knowledge of foods (e.g. 'All spices have a high sodium (salt) content') or food/nutrient-disease relationships (e.g. 'Anaemia can be caused by an iron deficiency'). All items were 
presented as 'agree or disagree' question type. An 'I don't know' option was also available for these items.

\section{Validity and reliability testing}

The validation of the nutrition knowledge questionnaire was achieved within the context of a larger study, which took place between February and August 2015. Biomarkers of fruit and vegetable intake were also measured but these analyses are beyond the scope of the present paper. Validation of the nutrition knowledge questionnaire was divided into three subsequent parts: (i) an expert panel evaluation; (ii) a pre-test; and (iii) a validation study.

\section{Expert panel evaluation}

Participants and procedures. An expert panel consisting of six members of the research team and including four nutrition researchers, an RD and a psychology researcher was formed to evaluate the nutrition knowledge questionnaire. The panel evaluated the questionnaire for content validity, either pen-and-paper or online.

Content validity. Content validity involves a rigorous assessment of the items to ensure representation of the construct, in this case nutrition knowledge. The panel evaluated the content validity of the questionnaire by commenting on the items and questionnaire format, and a content validity index was calculated for each item. The index used was based on four criteria, namely relevance ('not relevant' (=1) to 'very relevant' $(=4))$, clarity ('not clear' $(=1)$ to 'very clear' $(=4)$ ), simplicity ('not simple' (=1) to 'very simple' (=4)) and ambiguity ('doubtful' $(=1)$ to 'meaning is clear' $(=4)$ ). Each criterion was evaluated on a four-point scale. Experts rated each item of the questionnaire and the evaluations were combined to yield a percentage of content validity. The acceptable level was set as $>80 \%$ since this is generally considered to be the minimum value for adequate content validity ${ }^{(22)}$.

\section{Pre-test}

Participants and procedures. The pre-test sample consisted of thirty individuals recruited from an internal list of people willing to participate in clinical studies. They were asked to comment about the acceptability and understanding of the items. Fifteen RD were also recruited for the pre-test. Participants were men and women from the region of Quebec and had to be aged between 18 and 65 years old. Participants were required to have at least a minimal skill level in informatics since the questionnaire was completed on an online survey website.

Face validity. Face validity indicates whether the items seem to measure what the developers claim they measure $^{(22)}$. Face validity was assessed in the pre-test by asking participants to comment specifically on the ambiguity of the items and questionnaire. Participants commented using online forms and commented in a comments box after each item.
Construct validity. The assessment of construct validity ensures that the construct intended to be measured is indeed measured by the questionnaire. In the present study, construct validity was measured using scores from the pre-test participants and comparing them with scores obtained by the RD sample, with the latter group expected to perform higher than those having no nutrition qualifications. Student $t$ tests were used to compare results from both groups.

\section{Validation study}

Participants and procedures. Participants for the validation study were recruited using electronic messages sent to Laval University employees and students from over 500 study programmes, as well as via the electronic newsletter of the Institute of Nutrition and Functional Foods, which includes people outside the University's scope who had previously registered to receive the newsletter. The study sample included 150 participants with an equal number of men and women. Inclusion criteria were identical to those in the pretest. Participants suffering from conditions that affected intestinal absorption were excluded, since it could alter biomarker measurements. Following their recruitment in the study, participants came to the research centre in a fasting state (12 h) since blood samples were drawn for measuring biomarkers. Trained professionals measured height and weight and waist circumference according to standardized procedures ${ }^{(23)}$. Participants had to complete an $\mathrm{FFQ}^{(24)}$ on an online platform during their visit. Within a month after coming to the laboratory, eight questionnaires including the nutrition knowledge questionnaire were completed at home by the participants on the online interface. Questionnaires were assigned to each participants in a random order. Mean completion time of all questionnaires was approximately $40 \mathrm{~min}$. Following a two-week resting period, participants had another month to complete each questionnaire a second time (again in a random order) to assess test-retest reliability. Participants received a financial compensation of \$CAN 50 for their participation in the study.

Exploratory factor analysis. Exploratory factor analysis was performed on the items to verify that the number of factors predicted (two subscales: 'familiarity with CFG' and 'general nutrition knowledge') was accurate for the questionnaire. A scree plot was generated to evaluate the number of factors associated with the questionnaire. According to Cattell ${ }^{(25)}$, in a scree plot, the 'elbow' of the plot is a point below which factors explain relatively little variance and above which they explain more. Cattell advises to retain factors above said 'elbow' and reject factors below this point ${ }^{(25)}$. The number of factors - or number of subscales - of the questionnaire was obtained using this technique.

Covariance structure analysis. Covariance structure analysis was performed, using confirmatory factor analysis, on all items to verify $t$ values of the items, in order to identify which items loaded too weakly with the 
factor and thus had to be removed. The criterion for retaining an item was to obtain a $t$ value above 1.96 , which indicates that the item is significantly associated with the factor $(P=0.05)^{(26)}$.

Internal consistency. Cronbach's $\alpha$ coefficients were used to measure the consistency of responses at the first completion of the questionnaire. Cronbach's $\alpha$ coefficients are expected to be 0.7 or higher for a scale to be considered consistent ${ }^{(27)}$.

Test-retest reliability. As indicated above, participants in the validation study completed the questionnaire twice to evaluate test-retest reliability and the time interval between both completions varied for each participant. Pearson's correlations between the two completions were performed to assess reliability. Partial correlations were used to evaluate the association between scores on both completions while controlling for the effect of the time interval.

Concurrent validity. Concurrent validity was obtained by measuring the correspondence between the participants' nutrition knowledge score and their dietary intakes. Data from the FFQ were used to assess diet quality through a Canadian adaptation of Kennedy et al.'s Healthy Eating Index (HEI $)^{(28,29)}$. The tool was further adapted by the research team to match the recommendations of the most recent CFG according to sex and age ${ }^{(30)}$. The HEI is composed of ten components, each evaluated on 10 points; individuals receive 10 points if the criterion is met perfectly, 0 points if they fail to meet the criterion, and a proportional score if between the two extremes. Fruit and vegetable servings were grouped, to adapt the criteria to the CFG recommendation ${ }^{(28)}$. A maximum of 20 points is thus attributed for this group. Component scores are summed for a total score ranging between 0 and 100 (100 being the best score possible). Concurrent validity was assessed with correlation analyses between scores for the nutrition knowledge questionnaire and HEI scores. For the concurrent validity analyses, participants who reported implausible food intakes in the FFQ were excluded. To do so, the Outlier Labeling Rule was used, with a 2.2 interquartile range $(\mathrm{IQR})$ multiplier ${ }^{(31)}$. This technique uses the sample quartiles, Q1 and Q3, and labels as 'outliers' any observations below $\mathrm{Q} 1-k(\mathrm{IQR})$ or above $\mathrm{Q} 3+k(\mathrm{IQR})$, with $k=2 \cdot 2$. Outliers were identified for energy intake, as well as for each of the four groups of the CFG (i.e. Vegetables and fruits, Grain products, Milk and alternatives, Meat and alternatives). Concurrent validity was also assessed by measuring correspondence between the nutrition knowledge score and the intake of fruit and vegetables.

\section{Statistical analyses}

Statistical tests were two-sided and differences at $P<0.05$ were considered significant. All statistical analyses were performed using the statistical software package SAS $^{\circledR}$ Studio version 3.3 (C) 2012-2015).

\section{Results}

\section{Expert panel evaluation}

Content validity

Following the experts' evaluation of content validity, further modifications were made to the questionnaire. Calculation of the content validity index revealed an average of $88 \%$ for all items, with three items with indices under $80 \%$. However, with approval from the expert committee, one of the items that obtained a content validity of $75 \%$ was reworded instead of removed to alleviate ambiguity, as it was considered important to measure nutrition knowledge ('Vitamin and mineral supplements can act as substitutes equal to fruit' reworded as 'It is not necessary to eat fruit when you take vitamin and mineral supplements'). Two items were completely removed from the questionnaire because of low content validity index (50 and $75 \%$, respectively) and questionable relevance with the nutrition constructs to be measured, as well as higher ambiguity for the responders. These items, which had to be answered as either 'I agree' or 'I disagree', were the following: 'The glycaemic index classifies foods according to their effect on glucose blood level' and 'A balanced diet means eating all foods in equal amounts'. Furthermore, compilation of comments from the experts led to reformulation of two questions ('How many portions a day do you think CFG recommends, for an individual of your age and gender, for each of the following food groups' and 'How many CFG portions do you think are equivalent to the following quantities of food?').

\section{Pre-test}

In the pre-test, gender balance was different between the $\mathrm{RD}$ and the non-RD sample groups (93\% of RD were female $v .55 \%$ of non-RD). The higher percentage of female $\mathrm{RD}$ is representative of the Canadian population where $96 \%$ of RD were female in $2011^{(32)}$. The mean age of participants was 46 (SD 14) years old.

\section{Face validity}

Face validity of the questionnaire was assessed by participants from the pre-test, who formulated comments on ambiguity of the items and questionnaire. According to participants' comments, one item necessitated reformulating ('Drink enriched soya beverages if you do not drink milk' was reformulated as 'Enriched soya beverages can be consumed as a replacement for milk'). The introduction of the questionnaire was also reworded following comments from participants, to ensure they would answer with respect to their own knowledge and not feel pressured to give correct answers.

\section{Construct validity}

As shown in Table 1, when comparing the RD group with the non-RD group for construct validity, the RD group 
Table 1 Comparison of total nutrition knowledge scores between registered dietitian (RD; $n$ 15) and non-RD $(n 30)$ samples ${ }^{*}$ in the construct validity evaluation of a nutrition knowledge questionnaire for French Canadians

\begin{tabular}{|c|c|c|c|c|c|c|c|c|c|c|}
\hline & \multicolumn{4}{|c|}{ RD sample } & \multicolumn{4}{|c|}{ Non-RD sample } & \multicolumn{2}{|c|}{ Difference between group means } \\
\hline & Mean & SD & Min. & Max. & Mean & SD & Min. & Max. & Mean difference & $P$ value \\
\hline Total nutrition knowledge score (out of 24) & 21.5 & $2 \cdot 1$ & $18 \cdot 1$ & $24 \cdot 0$ & $15 \cdot 7$ & 3.0 & $5 \cdot 0$ & $20 \cdot 6$ & $5 \cdot 8$ & 0.0001 \\
\hline
\end{tabular}

Min., minimum; max., maximum.

${ }^{*}$ After removing items that were not loading properly, the mean score was still significantly higher for RD than for non-RD (12.2 (SD 1.2$)$ v. 9.3 (SD 1.80 out of 13.5 , respectively, $P<0.0001$ ).

scored significantly higher than the non-RD group $(21 \cdot 5$ (SD 2.1) v. $15 \cdot 7$ (sD 3.0) out of 24, $P<0 \cdot 001,23 \%$ difference in total score).

\section{Validation study}

In the validation study, two participants dropped out before completing the questionnaire and for the test-retest assessment, two more failed to perform the second completion. Participants were selected to include an equal number of men and women, but due to non-completion of questionnaires, the final sample included $50 \cdot 7 \%$ female and $49.3 \%$ male participants. The mean age of participants in the validation study was 47 (SD 13) years old and their mean BMI was 25.5 (SD 4.4$) \mathrm{kg} / \mathrm{m}^{2}$. Table 2 presents the sample characteristics in more detail.

\section{Exploratory factor analysis}

Following analysis of the scree plot from exploratory factor analysis, it was observed that the number of factors above the 'elbow', and thus the number of factors that should be considered in the questionnaire, was one. Therefore, in contrast to the a priori categorization of the questionnaire into two subscales ('familiarity with CFG' and 'general nutrition knowledge'), the analysis suggested that the questionnaire consisted in fact of only one global nutrition knowledge scale.

\section{Covariance structure analysis}

The $t$ values obtained for factor loading using covariance structure analysis ranged from $-0 \cdot 84$ to $7 \cdot 17$. Using this analysis, sixteen items were identified as loading too poorly with the nutrition knowledge factor. Therefore, these items were removed from the questionnaire. The online supplementary material presents all the items and their associated $t$ values.

\section{Internal consistency}

When considering the questionnaire after removal of the weakly loading items, internal consistency was adequate, with Cronbach's $\alpha$ coefficient of 0.73 . Note that this value was improved compared with prior analysis performed with all items, including those with a weak loading (Cronbach's $\alpha=0 \cdot 68$ ).
Table 2 Characteristics of participants $(n$ 148) in the validation study of a nutrition knowledge questionnaire for French Canadians

\begin{tabular}{|c|c|c|}
\hline & $n$ & $\%$ \\
\hline \multicolumn{3}{|l|}{ Gender } \\
\hline Male & 73 & $49 \cdot 3$ \\
\hline Female & 75 & $50 \cdot 7$ \\
\hline \multicolumn{3}{|l|}{ Age (years) } \\
\hline $18-34$ & 38 & $25 \cdot 7$ \\
\hline $35-49$ & 29 & $19 \cdot 6$ \\
\hline $50-65$ & 81 & 54.7 \\
\hline \multicolumn{3}{|l|}{ BMI category } \\
\hline Underweight $\left(0-18.4 \mathrm{~kg} / \mathrm{m}^{2}\right)$ & 4 & $2 \cdot 7$ \\
\hline Normal $\left(18.5-24.9 \mathrm{~kg} / \mathrm{m}^{2}\right)$ & 70 & $47 \cdot 3$ \\
\hline Overweight $\left(25.0-29.9 \mathrm{~kg} / \mathrm{m}^{2}\right)$ & 54 & $36 \cdot 5$ \\
\hline Obese $\left(>30.0 \mathrm{~kg} / \mathrm{m}^{2}\right)$ & 20 & 13.5 \\
\hline \multicolumn{3}{|l|}{ Income (\$CAN) } \\
\hline $0-19999$ & 8 & 5.4 \\
\hline 20000-39999 & 18 & $12 \cdot 2$ \\
\hline $40000-59999$ & 26 & $17 \cdot 6$ \\
\hline $60000-79999$ & 22 & $15 \cdot 9$ \\
\hline $80000-99999$ & 19 & $12 \cdot 9$ \\
\hline$\geq 100000$ & 44 & $32 \cdot 1$ \\
\hline No response & 11 & 7.4 \\
\hline \multicolumn{3}{|l|}{ Education } \\
\hline No education & 0 & 0.0 \\
\hline Primary school & 0 & 0.0 \\
\hline High school diploma or equivalent & 14 & 9.5 \\
\hline College graduate & 45 & 30.4 \\
\hline University graduate & 89 & $60 \cdot 1$ \\
\hline \multicolumn{3}{|l|}{ Race/ethnicity } \\
\hline African & 2 & 1.4 \\
\hline Native Americans & 1 & 0.7 \\
\hline Arabic & 2 & 1.4 \\
\hline Asian & 0 & 0.0 \\
\hline Caribbean & 0 & 0.0 \\
\hline Caucasian & 142 & $96 \cdot 0$ \\
\hline Latino & 1 & 0.7 \\
\hline No response & 0 & 0.0 \\
\hline \multicolumn{3}{|l|}{ Marital status } \\
\hline Single & 50 & 33.8 \\
\hline Married & 37 & $25 \cdot 0$ \\
\hline Common-law partner & 45 & 30.4 \\
\hline Separated & 4 & 2.7 \\
\hline Divorced & 11 & $7 \cdot 4$ \\
\hline Widowed & 0 & 0.0 \\
\hline No response & 1 & 0.7 \\
\hline \multicolumn{3}{|l|}{ Primary employment status } \\
\hline Student & 9 & $6 \cdot 2$ \\
\hline Employed full time & 85 & $58 \cdot 2$ \\
\hline Employed part time & 12 & 8.2 \\
\hline Unemployed & 3 & $2 \cdot 0$ \\
\hline Homemaker & 0 & 0.0 \\
\hline Retired & 32 & 21.9 \\
\hline Unable to work & 0 & 0.0 \\
\hline Other & 5 & 3.4 \\
\hline No response & 0 & 0.0 \\
\hline
\end{tabular}




\section{Test-retest reliability}

Average time between the two completions of the questionnaire was 40 (SD 12) d (range 15-110d). Pearson's correlation between both completions was moderate but significant for overall score $(r=0.59, P<0.0001)$. When adjusted for time interval between completions, partial correlation between both completions was stronger ( $r=0.72, P<0.0001)$. When the sample was split into two groups based on the median value of the time interval between both completions (i.e. 39.0 d), there was a stronger correlation between completions for values above $(r=0.74) v$. below the median value $(r=0.41)$.

\section{Concurrent validity}

The HEI was used as a proxy of diet quality to assess concurrent validity through correlations with the scores obtained for the nutrition knowledge questionnaire. For these analyses, ten participants (four women and six men) were excluded because of unrealistic food intakes, based on the Outlier Labeling Rules explained above. Correlation obtained for concurrent validity with HEI score was moderate $(r=0.39, P<0 \cdot 0001)$, as was the correlation with fruit and vegetable intake $(r=0.31, P=0.0002)$.

\section{Analyses according to BMI}

Although the aim of the study was to validate the questionnaire in a sample representing the whole population, we used the opportunity provided by the wide BMI range of our sample to conduct additional analyses within subgroups separated on the basis of BMI. Accordingly, some analyses were conducted within the subgroup of participants with a normal weight (i.e. BMI $<25 \mathrm{~kg} / \mathrm{m}^{2}, n 74$ ) as well as within a subgroup with $\mathrm{BMI} \geq 25 \mathrm{~kg} / \mathrm{m}^{2}$ (overweight and obese participants, $n$ 74). We did observe some variability in $t$ values obtained, but it did not have any major impact on the test-retest values $(r=0.64$ for participants with normal weight and $r=0.55$ for overweight/obese participants $v . r=0.59$ for overall sample). As for internal consistency, the value for participants with normal weight was somewhat lower than in the overall sample (0.61 v. 0.73$)$ and for overweight/obese participants, it was somewhat higher than in the overall sample $(0.78 v .0 .73)$.

\section{Discussion}

The purpose of the present study was to develop and validate a nutrition knowledge questionnaire for a FrenchCanadian population. The questionnaire was developed with the aim of examining knowledge of CFG guidelines, as well as more general nutrition knowledge, for a FrenchCanadian population. Items were designed or chosen for either their relevance with CFG or their assessment of nutrition knowledge in general, focusing on nutrients and on links between nutrition and health.
Face validity was useful to assess participants' understanding of the items and their comments, although they did not cause any major change to the questionnaire, improved the wording of questions that caused ambiguity, as seen in other studies ${ }^{(33,34)}$. To measure construct validity, the 'known-groups approach' was used, where the researcher tests the hypothesis that of two or more groups of participants, one group is expected to score higher on the construct of interest compared with another group $^{(22)}$. In this case, it was anticipated that RD would obtain higher scores than participants from the pre-test, who had no nutrition schooling background, and significant differences were indeed observed between both groups, indicating satisfactory construct validity. RD scored consistently higher on overall nutrition knowledge score (23\% difference). This validates the questionnaire's ability to distinguish between groups with different nutrition knowledge levels. Compared with other studies comparing a community sample with either final-year nutrition students or RD, the difference in scores observed in the present study was higher than for a nutrition knowledge questionnaire administered in an Australian sample (12\% difference, final-year dietetics students ${ }^{(11)}$ ) and also higher than for a knowledge questionnaire about salt for adults ( $17 \%$ difference, $\mathrm{RD}^{(35)}$ ), but lower than the GNKQ (35\% difference, final-year dietetics students ${ }^{(10)}$ ).

Assessment of internal consistency revealed adequate overall Cronbach's $\alpha(\alpha=0.73)$. Similar values have been obtained in other studies ${ }^{(11,19)}$. Although test-retest reliability was significant for the questionnaire, the Pearson's $r$ value obtained was not particularly high, at $0 \cdot 59$, compared with other studies ${ }^{(10,36,37)}$. However, the time interval between both completions was longer than observed in other studies, where generally two weeks separated completions ${ }^{(10-12,36-38)}$. In the present study, due to constraint linked with the context of the study, the time interval varied from 15 to $110 \mathrm{~d}$. According to the literature, more than two weeks could be enough to modify nutrition knowledge due to the constant flow of information in this domain ${ }^{(22)}$. The partial correlation analysis that controlled for time interval between completions led to an increased correlation coefficient. More specifically, it was found that the correlation between both completions was stronger when the time interval was longer. Therefore, the longer time interval between questionnaire completions in the present study compared with others is apparently not contributing to the relatively lower test-retest reliability observed. Moreover, it was observed that the mean score for participants was slightly higher in the second completion, which could be explained by a general improvement in nutrition knowledge of the participants. Another explanation could be that, although it was advised not to, some participants would have undertaken research following the first completion out of curiosity, and would therefore have improved their scores on the second completion of the 
questionnaire, which could have contributed to reduce the test-retest reliability. Results conducted in subgroups separated on the basis of BMI showed that values obtained in normal-weight participants were not exactly the same as those obtained in overweight/obese participants for internal consistency and test-retest values. These results demonstrate the importance of validating the questionnaire again in order to measure nutrition knowledge in a specific population. Concurrent validity was measured by examining the association between food intake and nutrition knowledge. Our results showed that participants with higher knowledge had better diet quality. In other studies, a significant correlation was also found between nutrition knowledge and adequate food intake; although the correlation observed in the present study was higher $^{(3,39)}$. Concurrent validity for fruit and vegetable consumption also revealed a significantly higher intake in participants with higher nutrition knowledge. Correlation between nutrition knowledge and fruit and vegetable intake was comparable to that observed by DicksonSpillman and Siegrist $(r=0.29$ for vegetables, $r=0.18$ for fruits) ${ }^{(39)}$ and Wardle et al. $(r=0 \cdot 36)^{(40)}$.

Some items were removed as they loaded too weakly with total nutrition knowledge score. When examining which items had been removed, some were identified as being representative of nutrition topics that are frequently discussed in the media and for which information is often contradictory. These items referred to topics such as milk consumption, the concept of food portions and the link between sugar and diabetes. These topics are often discussed in the media and different interest groups can disclose different information about them ${ }^{(41)}$. Individuals can be left with a feeling of confusion between those contradictory messages, between actual scientific nutrition research and the media's false interpretation of the conclusions $^{(42)}$. The weakly loading items that were concerned with those topics could be explained by the confusion created around them. In fact, this shows that even within individuals in the population with higher nutrition knowledge, who are more likely to be well informed, these themes can be unclear. Even in scientific literature, some authors disagree about topics such as dairy products and milk ${ }^{(43-45)}$. Therefore, the distinction between people with higher nutrition knowledge and lower nutrition knowledge could have been less pronounced when assessed using these items. Another important issue to consider is the ever-evolving aspect of nutrition and that the information conveyed changes in time. Nutrition knowledge questionnaires have to be adjusted when major changes occur in the scientific literature and validation of the questionnaire can be necessary to ensure that, with passing time, the chosen items are still valid and reliable.

The major strengths of the current study include the method of development and evaluation of the questionnaire, which was based on the steps proposed by
Parmenter and Wardle $\mathrm{W}^{(13)}$ in their report on nutrition knowledge measures. From generation of items to evaluation of the questionnaire, the report was used as a guide for questionnaire design and validation. Another strength is the large age and BMI range of the validation study sample. However, most participants were highly educated, which is not perfectly representative of the Canadian population. For the development of the questionnaire, it could have been interesting to begin with a larger item pool, which would have allowed stricter item difficulty cut-off points. Nevertheless, it must be mentioned that a short questionnaire was preferable for the present study, and thus the item pool did not have to be as large as for other questionnaires. Moreover, the study was conducted in a university facility, the Institute of Nutrition and Functional Foods, which is well known in the Quebec City vicinity for the studies it performs related to health and nutrition. Thus, there is a possibility that participants recruited for the study had a particular interest towards nutrition and possibly more nutrition knowledge. Also, it can be noted that the questionnaire has been validated in a French-Canadian population with specific cultural, geographical and sociodemographic characteristics, and thus it would be important to validate it again if used in another population.

\section{Conclusion}

The nutrition knowledge questionnaire developed for a French-Canadian population is a valid and reliable tool to assess nutrition knowledge and to discriminate between different knowledge levels. It should be noted that validity and reliability are acceptable but should be tested again if the questionnaire is to be used in other populations. This questionnaire could serve as a model for the development of similar tools in other populations, based on their local recommendations. For future adaptations of the questionnaire, the aspect of sustainability of diets could also be included, since it is now an important part of advocated diets apart from the healthy aspect.

\section{Acknowledgements}

Financial support: This work was supported by the Canadian Institutes of Health Research (grant number FHG129921). The Canadian Institutes of Health Research had no role in the design, analysis or writing of this article. Conflict of interest: None. Authorship: V.P., C.B., J.R., S.D., M.-C.V. and S.L. contributed in designing the study and formulating the research questions. M.B.-L., L.C. and E.C. were responsible for carrying out the study. M.B.-L. and E.C. analysed the data and wrote the article. All co-authors revised the final version of the paper. Ethics of buman subject participation: This study was conducted according to the guidelines laid down in the Declaration of 
Helsinki and all procedures involving human subjects were approved by the Research Ethics Committee at Laval University, Québec. Written informed consent was obtained from all participants.

\section{Supplementary material}

To view supplementary material for this article, please visit https://doi.org/10.1017/S1368980016003372

\section{References}

1. McLeroy KR, Bibeau D, Steckler A et al. (1988) An ecological perspective on health promotion programs. Health Educ $Q$ 15, 351-377.

2. Raine KD (2005) Determinants of healthy eating in Canada: an overview and synthesis. Can J Public Health 96, Suppl. 3, S8-S15.

3. Spronk I, Kullen C, Burdon C et al. (2014) Relationship between nutrition knowledge and dietary intake. BrJ Nutr 111, 1713-1726.

4. Staser KW, Zollinger TW, Saywell RM Jr. et al. (2011) Dietary behaviors associated with fruit and vegetable consumption, Marion County, Indiana, 2005. Prev Chronic Dis 8, A66.

5. Garriguet D (2009) Diet quality in Canada. Health Rep 20 , $41-52$.

6. Laz TH, Rahman M, Pohlmeier AM et al. (2015) Level of nutrition knowledge and its association with weight loss behaviors among low-income reproductive-age women. J Community Health 40, 542-548.

7. Cooper BR, Barale K, Funaiole A et al. (2016) Participant and household characteristics associated with graduation from the expanded food and nutrition education program. J Nutr Educ Behav 48, 453-460.e1.

8. Jennings L, Nepocatych S, Ketcham C et al. (2016) The effect of a summer camp intervention on the nutrition knowledge and dietary behavior of adolescent girls. Health Promot Pract 17, 521-529.

9. Pem D, Bhagwant S \& Jeewon R (2016) A pre and post survey to determine effectiveness of a dietitian-based nutrition education strategy on fruit and vegetable intake and energy intake among adults. Nutrients $\mathbf{8}, 127$.

10. Parmenter K \& Wardle J (1999) Development of a general nutrition knowledge questionnaire for adults. Eur J Clin Nutr 53, 298-308.

11. Hendrie GA, Cox DN \& Coveney J (2008) Validation of the general nutrition knowledge questionnaire in an Australian community sample. Nutr Diet 65, 72-77.

12. Alsaffar AA (2012) Validation of a general nutrition knowledge questionnaire in a Turkish student sample. Public Health Nutr 15, 2074-2085.

13. Parmenter K \& Wardle J (2000) Evaluation and design of nutrition knowledge measures. J Nutr Educ 32, 269-277.

14. Health Canada (2007) Eating Well with Canada's Food Guide. Ottawa, ON: Health Canada.

15. Mathe N, Van der Meer L, Agborsangaya CB et al. (2015) Prompted awareness and use of Eating Well with Canada's Food Guide: a population-based study. J Hum Nutr Diet $\mathbf{2 8}$, 64-71.

16. Anderson LC, Mah CL \& Sellen DW (2015) Eating well with Canada's food guide? Authoritative knowledge about food and health among newcomer mothers. Appetite $91,357-365$.

17. Lafave L (2008) Development of a Canadian Behaviour, Attitude and Nutrition Knowledge Survey (BANKS). https://www.google.co.uk/webhp?gws_rd=ssl ${ }^{*} \mathrm{q}=$ Lafave $+\mathrm{L}$ $+(2008)+$ Development+of+a+Canadian+Behaviour\%2C +Attitude+and+Nutrition+Knowledge+Survey+(BANKS) (accessed December 2016).

18. Canadian Council of Food and Nutrition (2009) Tracking Nutrition Trends: A 20-Year History. Mississauga, ON: Canadian Council of Food and Nutrition; available at https://www.cfdr.ca/Downloads/CCFN-docs/20-Years-ofTNT-(Sep12)_Final.aspx

19. Dickson-Spillmann M, Siegrist M \& Keller C (2011) Development and validation of a short, consumer-oriented nutrition knowledge questionnaire. Appetite 56, 617-620.

20. Rustad C \& Smith C (2013) Nutrition knowledge and associated behavior changes in a holistic, short-term nutrition education intervention with low-income women. $J$ Nutr Educ Behav 45, 490-498.

21. Pawlak R \& Colby S (2009) Benefits, barriers, self-efficacy and knowledge regarding healthy foods; perception of African Americans living in eastern North Carolina. Nutr Res Pract 3, 56-63.

22. Dilorio CK (2006) Measurement in Health Behavior: Methods for Research and Evaluation. San Francisco, CA: Jossey-Bass.

23. Callaway C, Chumlea W \& Bouchard C (1988) Standardization of anthropometric measurements. In The Airlie (VA) Consensus Conference, pp. 39-80 [T Lohman, A Roche and R Martorel, editors]. Champaign, IL: Human Kinetics Publishers.

24. Labonte ME, Cyr A, Baril-Gravel L et al. (2012) Validity and reproducibility of a web-based, self-administered food frequency questionnaire. Eur J Clin Nutr 66, 166-173.

25. Cattell RB (1966) The scree test for the number of factors. Multivariate Behav Res 1, 245-276.

26. Surh D (2006) Exploratory or Confirmatory Factor Analysis? Proceedings of the Annual Conference of SAS Users Group International. Cary, NC: SAS Institute.

27. Nunnally JC \& Bernstein IH (1994) Psychometric Theory, 3rd ed. New York: McGraw-Hill, Inc.

28. Dubois L, Girard M \& Bergeron N (2000) The choice of a diet quality indicator to evaluate the nutritional health of populations. Public Health Nutr 3, 357-365.

29. Kennedy ET, Ohls J, Carlson S et al. (1995) The Healthy Eating Index: design and applications. J Am Diet Assoc 95, 1103-1108.

30. Health Canada (2011) Eating Well with Canada's Food Guide - A Resource for Educators and Communicators. Ottawa, ON: Health Canada; available at Ottawa, ON: Health Canada.

31. Hoaglin DC \& Iglewicz B (1987) Fine-tuning some resistant rules for outlier labeling. J Am Stat Assoc 82, 1147-1149.

32. Statistique Canada (2013) Enquête nationale auprès des ménages de 2011 - Profession. Ottawa, ON: Statistique Canada.

33. Feren A, Torheim LE \& Lillegaard IT (2011) Development of a nutrition knowledge questionnaire for obese adults. Food Nutr Res 2011, 55.

34. Anderson AS, Bell A, Adamson A et al. (2002) A questionnaire assessment of nutrition knowledge - validity and reliability issues. Public Health Nutr 5, 497-503.

35. Sarmugam R, Worsley A \& Flood V (2014) Development and validation of a salt knowledge questionnaire. Public Health Nutr 17, 1061-1068.

36. Jones AM, Lamp C, Neelon M et al. (2015) Reliability and validity of nutrition knowledge questionnaire for adults. J Nutr Educ Behav 47, 69-74.

37. Deniz MS \& Alsaffar AA (2013) Assessing the validity and reliability of a questionnaire on dietary fibre-related knowledge in a Turkish student population. J Health Popul Nutr 31, 497-503.

38. de Pinho L, Moura PH, Silveira MF et al. (2013) Development and validity of a questionnaire to test the knowledge 
of primary care personnel regarding nutrition in obese adolescents. BMC Fam Pract 14, 102.

39. Dickson-Spillmann M \& Siegrist M (2011) Consumers' knowledge of healthy diets and its correlation with dietary behaviour. J Hum Nutr Diet 24, 54-60.

40. Wardle J, Parmenter K \& Waller J (2000) Nutrition knowledge and food intake. Appetite 34, 269-275.

41. Fitzgibbon M, Gans KM, Evans WD et al. (2007) Communicating healthy eating: lessons learned and future directions. J Nutr Educ Behav 39, 2 Suppl., S63-S71.
42. Miller GD, Cohen NL, Fulgoni VL et al. (2006) From nutrition scientist to nutrition communicator: why you should take the leap. Am J Clin Nutr 83, 1272-1275.

43. Markey O, Vasilopoulou D, Givens DI et al. (2014) Dairy and cardiovascular health: friend or foe? Nutr Bull 39, 161-171.

44. Jones PJH (2015) New health benefits of dairy products. Am J Clin Nutr 101, 249-250.

45. Agostoni C \& Turck D (2011) Is cow's milk harmful to a child's health? J Pediatric Gastroenterol Nutr 53, 594-600. 